\title{
Finite Element Analysis of Multi-Stage Deep Drawing for Forming Deep Rectangular Case with High Aspect Ratio
}

\author{
Shohei Kajikawa*, Tomoya Takasan ${ }^{\dagger}$, Takashi Kuboki ${ }^{\dagger}$, Kenji Tamaoki ${ }^{\dagger \dagger}$, \\ Katsuyuki Onishi ${ }^{\dagger \dagger \dagger}$, Akira Gunji ${ }^{\dagger \dagger \dagger}$ and Akira Yamauchi ${ }^{\dagger \dagger \dagger}$ \\ * Department of Mechanical and Intelligent Systems Engineering \\ The University of Electro-Communications \\ 1-5-1 Chofu Gaoka, Chofu-shi, Tokyo, 182-8585, Japan \\ E-mail: s.kajikawa@uec.ac.jp - Web page: http://www.uec.ac.jp/eng/ \\ ${ }^{\dagger}$ Department of Mechanical and Intelligent Systems Engineering \\ The University of Electro-Communications \\ 1-5-1 Chofu Gaoka, Chofu-shi, Tokyo, 182-8585, Japan \\ E-mail: takasan@mt.mce.uec.ac.jp, kuboki@mce.uec.ac.jp \\ it Tokyo Metropolitan Industrial Technology Research Institute \\ 1-20-20 Minamikamata, Ota-ku, Tokyo, 144-0035, Japan \\ Email: tamaoki.kenji@iri-tokyo.jp - Web page: http://www.iri-tokyo.jp/ \\ Ti† Yamauchi Engineering Co., Ltd. \\ 2327-2 Tana, Chuo-ku, Sagamihara-shi, Kanagawa, 252-0244, Japan \\ E-mail:k-ohnishi@y-eng.jp, a-gunji@y-eng.jp, a-yamauchi@y-eng.jp \\ Web page: http://yama-eng.com/
}

\begin{abstract}
This paper presents investigations on multi-stage deep drawing for producing a deep rectangular case with high aspect ratio, which is used for a battery case in an electric vehicle. It is important to form the case which wall thickness and height are as uniform as possible. The local thinning leads to a crack. If the wall height is not uniform due to an edge-wave, the volume of the scrap increases. These issues could be easy to occur with the increase in the aspect ratio. There are many research works about deep drawing $[1,2]$, but few research works concern the rectangular case with high aspect ratio.

This paper focuses the effect of a blank shape on a formability. When the blank corner radius is too small, a load become too large due to a large shrink flange deformation, and then the crack occurs. The blank shape also affects the edge-wave shape. In this study, the effect of the blank shape on the formability was investigated in order to form the case with the aspect ratio of 10.3 by four-stage deep drawing. The blank was a rectangular shape with elliptical corners. The finite element analysis was carried out under the conditions of various corner radius of the blank, and then the thickness distribution and the edge-wave shape were evaluated.

As a result of the first stage, the local thinning occurred at the wall when the blank corner radius was small, while the local thinning improved by increasing the blank corner radius. The thickness uniformity after the fourth stage was improved by preventing the local thinning in the first stage. In addition, the edge-wave became small by optimizing the blank corner shape. These results showed a fundamental guideline for determining the blank shape for forming the rectangular case with high aspect ratio.
\end{abstract}

\section{REFERENCES}

[1] M. Ahmetoglu, T.R. Broek, G. Kinzel and T. Altan, "Control of blank holder force to eliminate wrinkling and fracture in deep-drawing rectangular parts", CIRP Ann., 44, 247-250 (1995).

[2] Y. Horikoshi, T. Kuboki, M. Murata, K. Matsui and M. Tsubokura, "Die design for deep drawing with high-pressured water jet utilizing computer fluid dynamics based on Reynolds' equation", $J$. Mater. Process. Technol., 218, 99-106 (2015). 